\title{
STRESSES IN A CIRCULAR CYLINDER HAVING A SPHERICAL CAVITY UNDER TENSION*
}

\author{
BY \\ CHIH-BING LING \\ Aeronautical Research Laboratory, Taiwan, China
}

Introduction. The stresses in a large tension member having a spherical cavity have been investigated by several authors, including the present writer. ${ }^{1-4}$ In dealing with such a problem the surface of the member is commonly assumed to be infinitely distant from the cavity so that the presence of the surface produces no effect on the stresses in the neighborhood of the cavity. However, this assumption is no longer valid when the surface of the member is at a finite distance from tine cavity. The consideration of the effect of the surface near the cavity naturally increases the analytic complication of the problem.

In the present paper a solution for an infinite circular cylinder having a spherical cavity under axial tension will be given. The cavity is assumed to be symmetrically located within the cylinder so that the theory of symmetrical strain for solids of revolu$\operatorname{tion}^{5}$ can be applied. The solution is obtained by constructing a stress function which satisfies the boundary conditions on the surface of the cylinder as well as at its ends. The boundary conditions on the surface of the cavity are satisfied by adjusting the coefficients of superposition involved in the solution. Here the stress function is necessarily a biharmonic function. ${ }^{6}$ The method of solution is first described. Then, as an illustration, numerical examples are given for two different radii of the cavity. In particular, the maximum stresses in the cylinder are calculated and shown graphically.

Method of solution. Denote as usual the cylindrical and spherical coordinates of a point by $(r, \theta, z)$ and $(\rho, \phi, \theta)$ respectively. For convenience, $r, z$ and $\rho$ will be regarded as dimensionless quantities referring to a typical length $a$. They are connected with each other by

$$
z=\rho \cos \phi, \quad r=\rho \sin \phi .
$$

Consider an infinite circular cylinder of radius $a$ having a symmetrically located spherical cavity of radius $\lambda a$ as shown in Fig. 1. The axis of the cylinder will be taken

*Received April 15, 1954; revised manuscript received Dec. 29, 1954.

${ }^{1}$ R. V. Southwell and H. J. Gough, Concentration of stress in the neighborhood of a small spherical flaw, etc., Phil. Mag. 1, 71-97 (1926).

${ }^{2} \mathrm{~J}$. N. Goodier, Concentration of stress around spherical and cylindrical inclusions and flaws, Trans. ASME 55, 39-44 (1933).

${ }^{3} \mathrm{H}$. Neuber, Theory of notch stresses, Edwards Brothers, 1946, English translation, pp. 102-104.

${ }^{4} \mathrm{C}$. B. Ling and K. L. Yang, On symmetrical strain in solids of revolution in spherical coordinates, Trans. ASME 73, A367-371 (1951). Note that in Eq. [32] and [34], p. 370, the factor $\left(2 n^{2}+2 n-3+\nu\right)$ should be read as $\left(2 n^{2}-1+\nu\right)$.

${ }^{5}$ A. E. H. Love, Mathematical theory of elasticity, fourth edition, Dover Publications, 1944, pp. 274-277.

'A different method of solution in such a case based on Boussinesq's approach is to find two harmonic functions. See footnote ${ }^{3}$. 
as the axis of $z$ and the center of the cavity as the origin. In the absence of the cavity, a uniform axial tension of $T$ per unit area would be given by the stress function

$$
\chi_{0}=\frac{T a^{3}}{6(1+\sigma)}\left\{3 \sigma r^{2} z+(1-2 \sigma) z^{3}\right\},
$$

where $\sigma$ is Poisson's ratio. The method of satisfying the boundary conditions when the cavity is present is to construct two sets of biharmonic functions each of which gives no traction on the surface of the cylinder and at the same time gives no stress at $z$ infinity or the ends of the cylinder. These functions are combined linearly and added to $\chi_{0}$. The

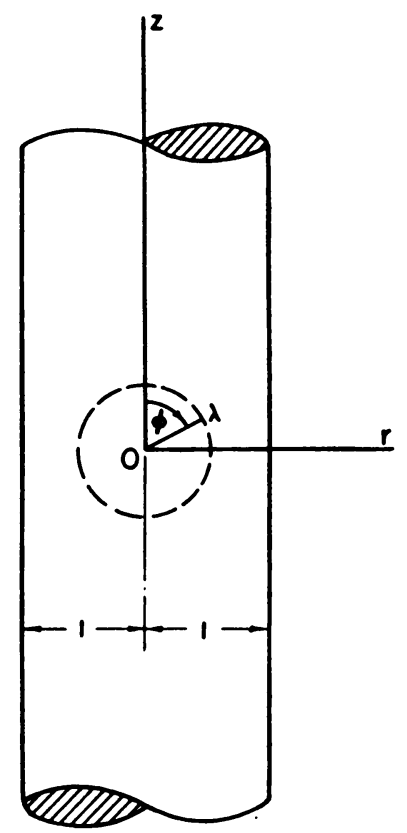

Fig. 1. The cylinder.

boundary conditions on the surface of the cavity are then satisfied by adjusting the coefficients of superposition attached to the functions. The sets of functions are derived by differentiation from two biharmonic functions each of which has a singularity at the origin.

Two sets of biharmonic functions. Consider a biharmonic function as follows:

$$
\chi_{1}=\frac{a^{3}}{2} \log \frac{1+\mu}{1-\mu}+a^{3} \int_{0}^{\infty}\left\{\psi_{1}(k) I_{0}(k r)+\psi_{2}(k) k r I_{1}(k r)\right\} \sin k z d k,
$$

where $I_{n}$ are modified Bessel functions of the first kind of order $n$ and $\psi_{n}$ are arbitrary functions, and

$$
\mu=\cos \phi .
$$

The first term of this function in fact represents a center of radial tension at the origin. The subsequent integral is added so as to annul the traction on the surface of 
the cylinder. This function gives the following normal and tangential stresses at $r=1$ or the surface of the cylinder.

$$
\begin{aligned}
{\left[\sigma_{r}\right]_{1}=} & \frac{1}{a^{3}}\left[\frac{\partial}{\partial z}\left\{\sigma\left(\frac{\partial^{2}}{\partial r^{2}}+\frac{1}{r} \frac{\partial}{\partial r}+\frac{\partial^{2}}{\partial z^{2}}\right)-\frac{\partial^{2}}{\partial r^{2}} \chi_{1}\right]_{r-1},\right. \\
= & \frac{1}{\left(1+z^{2}\right)^{3 / 2}}-\frac{3}{\left(1+z^{2}\right)^{5 / 2}}-\int_{0}^{\infty} k^{2}\left[\psi_{1}(k)\left\{k I_{0}(k)-I_{1}(k)\right\}\right. \\
& \left.\quad+\psi_{2}(k) k\left\{(1-2 \sigma) I_{0}(k)+k I_{1}(k)\right\}\right] \cos k z d k \\
{\left[\tau_{r z}\right]_{1} } & =\frac{1}{a^{3}}\left[\frac{\partial}{\partial r}\left\{(1-\sigma)\left(\frac{\partial^{2}}{\partial r^{2}}+\frac{1}{r} \frac{\partial}{\partial r}+\frac{\partial^{2}}{\partial z^{2}}\right)-\frac{\partial^{2}}{\partial z^{2}}\right\}_{1}\right]_{r-1}, \\
& =-\frac{3 z}{\left(1+z^{2}\right)^{5 / 2}}+\int_{0}^{\infty} k^{3}\left[\psi_{1}(k) I_{1}(k)+\psi_{2}(k)\left\{k I_{0}(k)+2(1-\sigma) I_{1}(k)\right\}\right] \sin k z d k .
\end{aligned}
$$

These stresses are annulled throughout the surface of the cylinder provided that by Fourier transforms,

$$
\begin{gathered}
\psi_{1}(k)\left\{k I_{0}(k)-I_{1}(k)\right\}+\psi_{2}(k) k\left\{(1-2 \sigma) I_{0}(k)+k I_{1}(k)\right\} \\
=\frac{2}{\pi k^{2}} \int_{0}^{\infty}\left\{\frac{1}{\left(1+z^{2}\right)^{3 / 2}}-\frac{3}{\left(1+z^{2}\right)^{5 / 2}}\right\} \cos k z d z, \\
\psi_{1}(k) I_{1}(k)+\psi_{2}(k)\left\{k I_{0}(k)+2(1-\sigma) I_{1}(k)\right\}=\frac{2}{\pi k^{3}} \int_{0}^{\infty} \frac{3 z \sin k z d z}{\left(1+z^{2}\right)^{5 / 2}}
\end{gathered}
$$

In view of the relation ${ }^{7}$,

$$
K_{n}(k)=\frac{1 \cdot 3 \cdot 5 \cdots(2 n-1)}{k^{n}} \int_{0}^{\infty} \frac{\cos k z d z}{\left(1+z^{2}\right)^{(2 n+1) / 2}},
$$

where $K_{n}$ are modified Bessel functions of the second kind of order $n$, we find

$$
\begin{aligned}
& \psi_{2}(k)=\frac{2}{\pi} \frac{I_{0}(k) K_{1}(k)+I_{1}(k) K_{0}(k)}{k^{2} I_{0}^{2}(k)-\left\{k^{2}+2(1-\sigma)\right\} I_{1}^{2}(k)}, \\
& \psi_{1}(k)=-\frac{2}{\pi} \frac{k I_{0}(k) K_{0}(k)+\left\{k^{2}+2(1-\sigma)\right\} I_{1}(k) K_{1}(k) / k}{k^{2} I_{0}^{2}(k)-\left\{k^{2}+2(1-\sigma)\right\} I_{1}^{2}(k)}-2(1-\sigma) \psi_{2}(k) .
\end{aligned}
$$

With these values of $\psi_{1}$ and $\psi_{2}$, it appears that the integral in (3) becomes divergent at the lower limit. However, the divergence can be removed by modifying the integral as follows:

$$
\chi_{1}=\frac{a^{3}}{2} \log \frac{1+\mu}{1-\mu}+a^{3} \int_{0}^{\infty}\left[\left\{\psi_{1}(k) I_{0}(k r)+\psi_{2}(k) k r I_{1}(k r)\right\} \sin k z+f_{1}(k) z\right] d k,
$$

where

$$
f_{1}(k)=\frac{4}{\pi(1+\sigma)}\left\{(2-\sigma) K_{0}(k)+\frac{3(1-\sigma)}{k} K_{1}(k)\right\}
$$

'G. N. Watson, Theory of Bessel functions, 2nd edition, 1944, p. 185, Cambridge University Press. Cf. the integral considered by Poisson and Malmstén. Also see p. 80 for the definition of $K_{n}$. 
such that the integral becomes convergent at the lower limit. This modification is permissible for it produces no effect on the stresses in the cylinder as well as on the convergence at the upper limit. It can be shown that the function $\chi_{1}$ thus obtained gives no stress at $z$ infinity or the ends of the cylinder.

It is obvious that successive differentiation with respect to $z$ gives functions with the desired properties on the surface of the cylinder as well as at $z$ infinity, but by symmetry odd derivatives must be excluded since they are even in $z$ and cannot enter into the required solution. The function $\chi_{1}$ itself may be included since it gives no unbalanced force at the origin. The set of functions is therefore

$$
\chi_{1}, \quad \frac{\partial^{2}}{\partial z^{2}} \chi_{1}, \quad \frac{\partial^{4}}{\partial z^{4}} \chi_{1}, \cdots .
$$

Again, consider a biharmonic function as follows:

$$
\chi_{2}=a^{3} \rho+a^{3} \int_{0}^{\infty}\left\{\psi_{3}(k) I_{0}(k r)+\psi_{4}(k) k r I_{1}(k r)\right\} \cos k z d k
$$

The first term of this function represents a concentrated force at the origin in the direction of $z$. This function gives the following normal and tangential stresses at $r=1$.

$$
\begin{aligned}
{\left[\sigma_{r}\right]_{1}=\frac{(1-2 \sigma) z}{\left(1+z^{2}\right)^{3 / 2}}-\frac{3 z}{\left(1+z^{2}\right)^{5 / 2}} } & +\int_{0}^{\infty} k^{2}\left[\psi_{3}(k)\left\{k I_{0}(k)-I_{1}(k)\right\}\right. \\
& \left.+\psi_{4}(k) k\left\{(1-2 \sigma) I_{0}(k)+k I_{1}(k)\right\}\right] \sin k z d k, \\
{\left[\tau_{r_{r}}\right]_{1}=-\frac{2(2-\sigma)}{\left(1+z^{2}\right)^{3 / 2}}+\frac{3}{\left(1+z^{2}\right)^{5 / 2}} } & +\int_{0}^{\infty} k^{3}\left[\psi_{3}(k) I_{1}(k)\right. \\
& \left.+\psi_{4}(k)\left\{k I_{0}(k)+2(1-\sigma) I_{1}(k)\right\}\right] \cos k z d k .
\end{aligned}
$$

These stresses are annulled throughout the surface of the cylinder provided that

$$
\begin{aligned}
\psi_{3}(k)\left\{k I_{0}(k)-I_{1}(k)\right\}+\psi_{4}(k) k\{ & \left.(1-2 \sigma) I_{0}(k)+k I_{1}(k)\right\} \\
& =-\frac{2}{\pi k^{2}} \int_{0}^{\infty}\left\{\frac{(1-2 \sigma) z}{\left(1+z^{2}\right)^{3 / 2}}-\frac{3 z}{\left(1+z^{2}\right)^{5 / 2}}\right\} \sin k z d z, \\
\psi_{3}(k) I_{1}(k)+\psi_{4}(k)\left\{k I_{0}(k)+2(1\right. & \left.-\sigma) I_{1}(k)\right\} \\
& =\frac{2}{\pi k^{3}} \int_{0}^{\infty}\left\{\frac{2(2-\sigma)}{\left(1+z^{2}\right)^{3 / 2}}-\frac{3}{\left(1+z^{2}\right)^{5 / 2}}\right\} \cos k z d z
\end{aligned}
$$

Similarly, we find in terms of $\psi_{1}$ and $\psi_{2}$,

$$
\begin{aligned}
& \psi_{3}(k)=\left\{k^{2}-2(1-\sigma)(1-2 \sigma)\right\} \psi_{2}(k) / k, \\
& \psi_{4}(k)=\left\{\psi_{1}(k)+4(1-\sigma) \psi_{2}(k)\right\} / k .
\end{aligned}
$$

In order to render the integral convergent at the lower limit, the function $\chi_{2}$ is to be modified as follows:

$$
\begin{aligned}
\chi_{2}=a^{3} \rho+a^{3} \int_{0}^{\infty}\left[\left\{\psi_{3}(k) I_{0}(k r)+\psi_{4}(k) k r I_{1}(k r)\right\} \cos k z\right. & \\
& \left.+f_{2}(k) r^{2}+f_{3}(k)\left(1-\frac{1}{2} k^{2} z^{2}\right)\right] d k,
\end{aligned}
$$


where

$$
\begin{aligned}
& f_{2}(k)=\frac{2}{\pi(1+\sigma)}\left\{\sigma K_{0}(k)-\frac{1-\sigma}{k} K_{1}(k)\right\}, \\
& f_{3}(k)=\frac{4(1-\sigma)(1-2 \sigma)}{\pi(1+\sigma) k^{2}}\left\{K_{0}(k)+\frac{2}{k} K_{1}(k)\right\} .
\end{aligned}
$$

It can be shown that the derivative of the function $\chi_{2}$ with respect to $z$ gives no stress at $z$ infinity.

It is equally obvious that odd derivatives of $\chi_{2}$ with respect to $z$ also gives functions with the desired properties. The set of functions is therefore

$$
\frac{\partial}{\partial z} \chi_{2}, \frac{\partial^{3}}{\partial z^{3}} \chi_{2}, \frac{\partial^{5}}{\partial z^{5}} \chi_{2}, \cdots
$$

Since the use of any constant multiplier does not affect the desired properties, we may write the two sets of functions as follows:

$$
\begin{gathered}
\omega_{0}=-\chi_{1}, \quad \omega_{2 s}=-\frac{1}{(2 s) !} \frac{\partial^{2 s}}{\partial z^{2 s}} \chi_{1}, \quad(s \geq 1), \\
\omega_{2 s}^{\prime}=-\frac{1}{(2 s+1) !} \frac{\partial^{2 s+1}}{\partial z^{2 s+1}} \chi_{2}, \quad(s \geq 0) .
\end{gathered}
$$

In expressing the functions in terms of spherical coordinates, the following relations are useful ${ }^{8}$.

$$
\begin{aligned}
& I_{0}(k r) \sin k z=\sum_{n=0}^{\infty}(-1)^{n} \frac{(k \rho)^{2 n+1}}{(2 n+1) !} P_{2 n+1}(\mu), \\
& k r I_{1}(k r) \sin k z=\sum_{n=0}^{\infty}(-1)^{n} \frac{(k \rho)^{2 n+1}}{(2 n+1) !}\left\{\frac{2 n(2 n+1)}{4 n+1}+\frac{(k \rho)^{2}}{4 n+5}\right\} P_{2 n+1}(\mu), \\
& \frac{\partial^{2 s}}{\partial z^{2 s}} \log \frac{1+\mu}{1-\mu}=-\frac{2(2 s-1) !}{\rho^{2 s}} P_{2 s-1}(\mu), \\
& \frac{\partial^{2 s+1} \rho}{\partial z^{2 s+1}}=-\frac{(2 s+1) !}{(4 s+1) \rho^{2 s}}\left\{P_{2 s-1}(\mu)-P_{2 s+1}(\mu)\right\}
\end{aligned}
$$

and in particular

$$
\frac{\partial \rho}{\partial z}=P_{1}(\mu)
$$

where $P_{n}$ are Legendre functions of the first kind of order $n$. Consequently, we have

$$
\begin{aligned}
& \omega_{0}=-\frac{a^{3}}{2} \log \frac{1+\mu}{1-\mu}+a^{3} \sum_{n=0}^{\infty}\left({ }^{2 n} \alpha_{0}+{ }^{2 n} \gamma_{0} \rho^{2}\right) \rho^{2 n+1} P_{2 n+1}(\mu), \\
& \omega_{2 s}=\frac{a^{3} P_{2 s-1}(\mu)}{2 s \rho}+a^{3} \sum_{n=0}^{\infty}\left({ }^{2 n} \alpha_{2 s}+{ }^{2 n} \gamma_{2 s} \rho^{2}\right) \rho^{2 n+1} P_{2 n+1}(\mu)
\end{aligned}
$$

${ }^{8}$ For the first relation, cf. Ex. 63, p. 362, T. M. MacRobert, Spherical harmonics, 2nd edition, 1947, Methuen. For the last two relations, see Exs. 21-22, p. 332, E. T. Whittaker and G. N. Watson, Modern analysis, 1927, Cambridge University Press; or see formula 36, p. 105, E. W. Hobson, Theory of spherical and ellipsoidal harmonics, 1931, Cambridge University Press. 
and

where

$$
\begin{aligned}
\omega_{0}^{\prime} & =-a^{3} P_{1}(\mu)+a^{3} \sum_{n=0}^{\infty}\left({ }^{2 n} \beta_{0}+{ }^{2 n} \delta_{0} \rho^{2}\right) \rho^{2 n+1} P_{2 n+1}(\mu), \\
\omega_{2 s}^{\prime} & =a^{3} \frac{P_{2 s-1}(\mu)-P_{2 s+1}(\mu)}{(4 s+1) \rho^{2 s}}+a^{3} \sum_{n=0}^{\infty}\left({ }^{2 n} \beta_{2 s}+{ }^{2 n} \delta_{2 s} \rho^{2}\right) \rho^{2 n+1} P_{2 n+1}(\mu),
\end{aligned}
$$

$$
\begin{aligned}
& { }^{2 n} \alpha_{2 s}=-\frac{(-1)^{n+s}}{(2 n) !(2 s) !} \int_{0}^{\infty}\left\{\frac{\psi_{1}(k)}{2 n+1}+\frac{2 n \psi_{2}(k)}{4 n+1}\right\} k^{2 n+2 s+1} d k \\
& { }^{2 n} \beta_{2 s}=\frac{(-1)^{n+s}}{(2 n) !(2 s+1) !} \int_{0}^{\infty}\left\{\frac{\psi_{3}(k)}{2 n+1}+\frac{2 n \psi_{4}(k)}{4 n+1}\right\} k^{2 n+2 s+2} d k \\
& { }^{2 n} \gamma_{2 s}=-\frac{(-1)^{n+s}}{(4 n+5)(2 n+1) !(2 s) !} \int_{0}^{\infty} \psi_{2}(k) k^{2 n+2 s+3} d k \\
& { }^{2 n} \delta_{2 s}=\frac{(-1)^{n+s}}{(4 n+5)(2 n+1) !(2 s+1) !} \int_{0}^{\infty} \psi_{4}(k) k^{2 n+2 s+4} d k
\end{aligned}
$$

and in particular

$$
\begin{aligned}
& { }^{0} \alpha_{0}=-\int_{0}^{\infty}\left\{k \psi_{1}(k)+f_{1}(k)\right\} d k, \\
& { }^{0} \beta_{0}=\int_{0}^{\infty} k^{2}\left\{\psi_{3}(k)+f_{3}(k)\right\} d k .
\end{aligned}
$$

The stress function. Now, construct the required stress function $\chi$ as follows:

$$
\chi=\chi_{0}+T \sum_{s=0}^{\infty}\left(A_{2 s} \omega_{2 s}+B_{2 s} \omega_{2 s}^{\prime}\right)
$$

where $A_{2}$, and $B_{2}$, aro coefficients of superposition to be determined from the remaining boundary conditions on the surface of the cavity; the factor $T$ being introduced to render the coefficients dimensionless. The function $\chi_{0}$ has been given in (2).

It is evident that the stress function thus constructed gives a uniform axial tension of $T$ per unit area at both ends of the cylinder and at the same time gives no traction on the surface of the cylinder. Its expression in spherical coordinates is readily found as follows:

$$
\begin{aligned}
x=\frac{T a^{3} \rho^{3}}{30(1+\sigma)}\left\{3 P_{1}(\mu)+2(1-\right. & \left.5 \sigma) P_{3}(\mu)\right\}-\frac{1}{2} T a^{3} A_{0} \log \frac{1+\mu}{1-\mu} \\
& +T a^{3} \sum_{n=1}^{\infty}\left(\frac{A_{2 n}}{2 n}+\frac{B_{2 n}}{4 n+1}-\frac{B_{2 n-2} \rho^{2}}{4 n-3}\right) \frac{P_{2 n-1}(\mu)}{\rho^{2 n}} \\
& +T a^{3} \sum_{n=0}^{\infty}\left(C_{2 n}+D_{2 n} \rho^{2}\right) \rho^{2 n+1} P_{2 n+1}(\mu)
\end{aligned}
$$

where

$$
\begin{aligned}
& C_{2 n}=\sum_{s=0}^{\infty}\left({ }^{2 n} \alpha_{2 s} A_{2,}+{ }^{2 n} \beta_{2 s} B_{2 s}\right), \\
& D_{2 n}=\sum_{i=0}^{\infty}\left({ }^{2 n} \gamma_{2 s} A_{2 s}+{ }^{2 n} \delta_{2 s} B_{2 s}\right) .
\end{aligned}
$$


This function gives the following normal and tangential stresses ${ }^{9}$

$$
\begin{aligned}
\sigma_{\rho}= & \frac{1}{a^{3}}\left\{(2-\sigma) \mu \frac{\partial}{\partial \rho}+\frac{\sigma\left(1-\mu^{2}\right)}{\rho} \frac{\partial}{\partial \mu}\right\} \nabla^{2} \chi-\frac{1}{a^{3}} \frac{\partial^{2}}{\partial \rho^{2}}\left(\mu \frac{\partial}{\partial \rho}+\frac{1-\mu^{2}}{\rho} \frac{\partial}{\partial \mu}\right) \chi \\
= & \frac{1}{3} T+\frac{2}{3} T P_{2}(\mu)+T \sum_{n=0}^{\infty}\left[\left\{\frac{(2 n+1)(2 n+2)}{\rho^{2}} A_{2 n}\right.\right. \\
& \left.+\frac{(2 n+1)(2 n+2)(2 n-2+4 \sigma)}{(4 n+3) \rho^{2}} B_{2 n}-\frac{4 n\left(2 n^{2}+3 n-\sigma\right)}{4 n-1} B_{2 n-2}\right\} \frac{1}{\rho^{2 n+1}} \\
& -\left\{\frac{2 n(2 n-1)(2 n+1)}{\rho^{2}} C_{2 n}-\frac{4 n(4 n-2)(3 n-4 n \sigma+1-\sigma)}{(4 n-1) \rho^{2}} D_{2 n-2}\right. \\
& \left.\left.+\frac{(4 n+2)(4 n+5)\left(2 n^{2}-n-1-\sigma\right)}{4 n+3} D_{2 n}\right\} \rho^{2 n}\right] P_{2 n}(\mu), \\
\tau_{\rho \phi}= & \frac{\left(1-\mu^{2}\right)^{1 / 2}}{a^{3}}\left\{-(1-\sigma)\left(\frac{\partial}{\partial \rho}+\frac{\mu}{\rho} \frac{\partial}{\partial \mu}\right) \nabla^{2} \chi+\frac{\partial}{\partial \rho}\left(\frac{1}{\rho} \frac{\partial}{\partial \mu}\right)\left(\mu \frac{\partial}{\partial \rho}+\frac{1-\mu^{2}}{\rho} \frac{\partial}{\partial \mu}\right) \chi\right\} \\
= & -\frac{1}{3} T\left(1-\mu^{2}\right)^{1 / 2} P_{2}^{\prime}(\mu)+T\left(1-\mu^{2}\right)^{1 / 2} \sum_{n=1}^{\infty}\left[\left\{\frac{2 n+2}{\rho^{2}} A_{2 n}\right.\right. \\
& \left.+\frac{(2 n+2)(2 n-2+4 \sigma)}{(4 n+3) \rho^{2}} B_{2 n}-\frac{2\left(2 n^{2}-1+\sigma\right)}{4 n-1} B_{2 n-2}\right\} \frac{1}{\rho^{2 n+1}} \\
& +\left\{\frac{(2 n-1)(2 n+1)}{\rho^{2}} C_{2 n}-\frac{(4 n-2)(3 n-4 n \sigma+1-\sigma)}{(4 n-1) \rho^{2}} D_{2 n-2}\right. \\
& \left.\left.+\frac{(4 n+5)\left(4 n^{2}+4 n-1+2 \sigma\right)}{4 n+3} D_{2 n}\right\} \rho^{2 n}\right] P_{2 n}^{\prime}(\mu) .
\end{aligned}
$$

Note that in spherical coordinates,

$$
\nabla^{2}=\frac{1}{\rho^{2}} \frac{\partial}{\partial \rho}\left(\rho^{2} \frac{\partial}{\partial \rho}\right)+\frac{1}{\rho^{2}} \frac{\partial}{\partial \mu}\left\{\left(1-\mu^{2}\right) \frac{\partial}{\partial \mu}\right\} .
$$

The remaining boundary conditions to be satisfied are that on the surface of the cavity the normal and tangential stresses vanish identically. By inserting $\rho=\lambda$ into (25) and (26) and equating separately the coefficient of each Legendre function or its derivative to zero, a system of linear equations is obtained. The system of equations may be replaced by the following system

$$
\begin{aligned}
A_{2 n}^{\prime}=-\frac{1}{3} \delta_{0, n}+ & \frac{4}{7-5 \sigma} \delta_{1, n}+2 n(n+1)(4 n-1) C_{2 n}^{\prime} \\
& \quad+2(4 n+1)\left(4 n^{4}+4 n^{3}-n^{2}-n+1-\sigma^{2}\right) D_{2 n}^{\prime}, \quad(n \geq 0), \\
B_{2 n-2}^{\prime}= & \frac{5}{3(7-5 \sigma)} \delta_{1, n}+(4 n+1) C_{2 n}^{\prime}+(2 n-1)(2 n+1)(4 n+3) D_{2 n}^{\prime}, \quad(n \geq 1),
\end{aligned}
$$

${ }^{\circ} \mathrm{Cf}$. footnote for the formulas of stresses in spherical coordinates. 
where $\delta_{m n}=1$ or 0 , according as $m=n$ or $m \neq n$, and

$$
\begin{aligned}
& A_{2 n}^{\prime}=\frac{2 n+2}{\lambda^{2 n+3}} A_{2 n}+\frac{(2 n+2)(2 n-2+4 \sigma)}{(4 n+3) \lambda^{2 n+3}} B_{2 n}, \\
& B_{2 n}^{\prime}=\frac{2}{(4 n+3) \lambda^{2 n+3}} B_{2 n}, \\
& C_{2 n}^{\prime}=-\frac{(2 n-1)(2 n+1) \lambda^{2 n-2}}{4 n^{2}+2 n-4 n \sigma+1-\sigma} C_{2 n} \\
& \quad \quad+\frac{4(2 n-1)(3 n-4 n \sigma+1-\sigma) \lambda^{2 n-2}}{(4 n-1)\left(4 n^{2}+2 n-4 n \sigma+1-\sigma\right)} D_{2 n-2}, \\
& D_{2 n}^{\prime}=-\frac{(4 n+5) \lambda^{2 n}}{(4 n+3)\left(4 n^{2}+2 n-4 n \sigma+1-\sigma\right)} D_{2 n} .
\end{aligned}
$$

A formal solution by successive approximations is as follows: Write, for $n \geq 0$,

where

$$
A_{2 n}^{\prime}=\sum_{p=0}^{\infty} A_{2 n}^{\prime(p)}, \quad B_{2 n}^{\prime}=\sum_{p=0}^{\infty} B_{2 n}^{\prime(p)}
$$

$$
\begin{aligned}
& A_{2 n}^{\prime(0)}=-\frac{1}{3} \delta_{0, n}+\frac{4}{7-5 \sigma} \delta_{1, n}, \\
& B_{2 n}^{\prime(0)}=\frac{5}{3(7-5 \sigma)} \delta_{0, n}
\end{aligned}
$$

and by iteration,

$$
\begin{aligned}
& A_{2 n}^{\prime(p)}=2 n(n+1)(4 n-1) C_{2 n}^{\prime(p-1)} \\
& \quad+2(4 n+1)\left(4 n^{4}+4 n^{3}-n^{2}-n+1-\sigma^{2}\right) D_{2 n}^{\prime(p-1)}, \quad(n \geq 0), \\
& B_{2 n-2}^{\prime(p)}=(4 n+1) C_{2 n}^{\prime(p-1)}+(2 n-1)(2 n+1)(4 n+3) D_{2 n}^{\prime(p-1)},
\end{aligned}
$$

in which $C_{2 n}^{(p-1)}$ and $D_{2 n}^{(p-1)}$ are computed from $A_{2 n}^{(p-1)}$ and $B_{2 n}^{(p-1)}$ by means of (29) and (24).

Naturally, the foregoing method of solving the linear equations is valid as long as the two series in (30) are both convergent. From physical consideration alone, it seems likely that there will be convergence if $\lambda$ is less than unity. An analytic proof of convergence may be established by means of the method used by Howland ${ }^{10}$ and Knight. ${ }^{11}$ However, for the sake of brevity no further detail will be given here. The solution will be illustrated by numerical examples which follow.

Numerical examples. Numerical examples will be given for the cases $\lambda=\frac{1}{2}$ and $\frac{1}{4}$. The coefficients in (21) are first computed and shown in Table 1, in which the integrals are integrated numerically from Gregory's formula ${ }^{12}$ with the aid of tables of Bessel functions. Here the Poisson's ratio $\sigma$ is taken as 0.3 . Note that ${ }^{\circ} \alpha_{2}$, and ${ }^{0} \beta_{2}$, are not

${ }^{10}$ R. C. J. Howland, Stresses in a plate containing an infinite row of holes, Proc. Roy. Soc. A148, 471-491, 1935.

11R. C. Knight, On the stresses in a perforated strip, Quart. J. Math., Oxford, 5, 255-268, 1934.

${ }^{12} \mathrm{E}$. T. Whittaker and G. Robinson, Calculus of observations, 4th ed., 1948, Blackie, p. 143. 
TABLE 1

\begin{tabular}{|c|c|c|c|c|c|c|c|}
\hline Coeff. & 28 & $2 n=0$ & $2 n=2$ & $2 n=4$ & $2 n=6$ & $2 n=8$ & $2 n=10$ \\
\hline${ }^{2 n} \alpha_{2}$ & $\begin{array}{r}0 \\
2 \\
4 \\
6 \\
8 \\
10\end{array}$ & $\begin{array}{l}- \\
- \\
- \\
-\end{array}$ & $\begin{array}{r}-0.50722 \\
0.90856 \\
-0.73705 \\
0.42356 \\
-0.20102 \\
0.08479\end{array}$ & $\begin{array}{r}0.058547 \\
-0.32478 \\
0.50010 \\
-0.46333 \\
0.32370 \\
-0.18884\end{array}$ & $\begin{array}{c}-0.0099477 \\
0.10476 \\
-0.26061 \\
0.35548 \\
-0.34379 \\
0.26537\end{array}$ & $\begin{array}{c}0.0018554 \\
-0.031758 \\
0.11653 \\
-0.22024 \\
0.28190 \\
-0.27813\end{array}$ & $\begin{array}{c}-0.00036549 \\
0.0092718 \\
-0.047229 \\
0.11819 \\
-0.19336 \\
0.23762\end{array}$ \\
\hline${ }^{2 n} \beta_{2 。}$ & $\begin{array}{r}0 \\
2 \\
4 \\
6 \\
8 \\
10\end{array}$ & $\begin{array}{l}- \\
- \\
- \\
-\end{array}$ & $\begin{array}{r}-0.42035 \\
0.54584 \\
-0.40438 \\
0.22351 \\
-0.10406 \\
0.04351\end{array}$ & $\begin{array}{r}0.12257 \\
-0.31634 \\
0.38274 \\
-0.31586 \\
0.20626 \\
-0.11520\end{array}$ & $\begin{array}{c}-0.032782 \\
0.14157 \\
-0.25723 \\
0.29768 \\
-0.26027 \\
0.18784\end{array}$ & $\begin{array}{c}0.0084192 \\
-0.055231 \\
0.14117 \\
-0.21899 \\
0.24757 \\
-0.22390\end{array}$ & $\begin{array}{c}-0.0021294 \\
0.019772 \\
-0.067883 \\
0.13621 \\
-0.19309 \\
0.21446\end{array}$ \\
\hline${ }^{2 n} \gamma_{2 。}$ & $\begin{array}{r}0 \\
2 \\
4 \\
6 \\
8 \\
10\end{array}$ & $\begin{array}{r}-0.35018 \\
0.37928 \\
-0.22980 \\
0.10584 \\
-0.04185 \\
0.01509\end{array}$ & $\begin{array}{r}0.070237 \\
-0.25533 \\
0.29397 \\
-0.21698 \\
0.12579 \\
-0.06258\end{array}$ & $\begin{array}{r}-0.017677 \\
0.12212 \\
-0.22533 \\
0.24384 \\
-0.19497 \\
0.12819\end{array}$ & $\begin{array}{c}0.0044470 \\
-0.049231 \\
0.13319 \\
-0.19880 \\
0.21005 \\
-0.17653\end{array}$ & $\begin{array}{c}-0.0011071 \\
0.017970 \\
-0.067055 \\
0.13226 \\
-0.17863 \\
0.18655\end{array}$ & $\begin{array}{c}0.00027445 \\
-0.0061446 \\
0.030298 \\
-0.076390 \\
0.12821 \\
-0.16314\end{array}$ \\
\hline${ }^{2 n} \delta_{28}$ & $\begin{array}{r}0 \\
2 \\
4 \\
6 \\
8 \\
10\end{array}$ & $\begin{array}{r}-0.048364 \\
0.16114 \\
-0.10336 \\
0.04842 \\
-0.01938 \\
0.00705\end{array}$ & $\begin{array}{r}0.089523 \\
-0.19141 \\
0.18829 \\
-0.12918 \\
0.07185 \\
-0.03486\end{array}$ & $\begin{array}{r}-0.039753 \\
0.13036 \\
-0.18781 \\
0.17907 \\
-0.13274 \\
0.08297\end{array}$ & $\begin{array}{c}0.014241 \\
-0.068389 \\
0.13694 \\
-0.17402 \\
0.16617 \\
-0.13012\end{array}$ & $\begin{array}{c}-0.0046136 \\
0.030793 \\
-0.082175 \\
0.13451 \\
-0.16093 \\
0.15450\end{array}$ & $\begin{array}{c}0.0014109 \\
-0.012550 \\
0.043140 \\
-0.088482 \\
0.12978 \\
-0.14990\end{array}$ \\
\hline
\end{tabular}

TABLE 2

\begin{tabular}{|c|c|c|c|c|c|}
\hline$\lambda$ & $2 n$ & $A_{2 n}^{\prime}$ & $B_{2 n}^{\prime}$ & $C_{2 n}^{\prime}$ & $D_{2 n}^{\prime}$ \\
\hline$\frac{1}{2}$ & $\begin{array}{r}0 \\
2 \\
4 \\
6 \\
8 \\
10\end{array}$ & $\begin{array}{r}-3.3487 \times 10^{-1} \\
8.4785 \times 10^{-1} \\
-5.563 \times 10^{-2} \\
1.072 \times 10^{-2} \\
-1.380 \times 10^{-3} \\
1.442 \times 10^{-4}\end{array}$ & $\begin{array}{r}3.5515 \times 10^{-1} \\
-6.037 \times 10^{-3} \\
5.318 \times 10^{-4} \\
-3.931 \times 10^{-5} \\
2.669 \times 10^{-6} \\
\ldots \ldots \ldots\end{array}$ & $\begin{array}{r}- \\
1.144 \times 10^{-2} \\
-8.070 \times 10^{-4} \\
5.261 \times 10^{-6} \\
-3.204 \times 10^{-6} \\
1.913 \times 10^{-7}\end{array}$ & $\begin{array}{r}-8.456 \times 10^{-4} \\
-2.411 \times 10^{-6} \\
7.428 \times 10^{-6} \\
-2.897 \times 10^{-7} \\
1.267 \times 10^{-8} \\
-5.920 \times 10^{-10}\end{array}$ \\
\hline$\frac{z}{2}$ & $\begin{array}{r}0 \\
2 \\
4 \\
6 \\
8 \\
10\end{array}$ & $\begin{array}{r}-3.3312 \times 10^{-1} \\
7.4303 \times 10^{-1} \\
-2.125 \times 10^{-3} \\
1.145 \times 10^{-4} \\
-4.135 \times 10^{-6} \\
1.223 \times 10^{-7}\end{array}$ & $\begin{array}{r}3.0966 \times 10^{-1} \\
-2.283 \times 10^{-4} \\
5.645 \times 10^{-6} \\
-1.172 \times 10^{-7} \\
2.240 \times 10^{-9} \\
\ldots \ldots \ldots\end{array}$ & $\begin{array}{r}- \\
1.360 \times 10^{-3} \\
-2.655 \times 10^{-5} \\
4.604 \times 10^{-7} \\
-7.412 \times 10^{-9} \\
1.163 \times 10^{-10}\end{array}$ & $\begin{aligned}+1.158 & \times 10^{-4} \\
-8.133 & \times 10^{-6} \\
6.424 & \times 10^{-8} \\
-6.475 & \times 10^{-10} \\
7.329 & \times 10^{-12} \\
-8.861 & \times 10^{-14}\end{aligned}$ \\
\hline
\end{tabular}


needed in computation for they are the coefficients of the trivial term $\rho P_{1}(\mu)$ or $z$. The coefficients $A_{2 n}^{\prime}, B_{2 n}^{\prime}, C_{2 n}^{\prime}$ and $D_{2 n}^{\prime}$ are then computed by the method of successive approximations for $\lambda=\frac{1}{2}$ and $\frac{1}{4}$ respectively. The results are shown in Table 2 . In the case $\lambda=\frac{1}{2}$ the computation has been carried out up to the fifth approximation. The convergence appears to be more rapid when $\lambda$ is smaller. The coefficients are readily converted to $A_{2 n}, B_{2 n}, C_{2 n}$ and $D_{2 n}$ by means of (29).

It is now rather straightforward to compute the stress at any point in the cylinder. The most important one, however, is the maximum stress occurring at the surface of the cavity across the minimum section where $(\rho, \mu)=(\lambda, 0)$. This stress is given by

$$
\begin{array}{r}
\max \sigma_{\phi}=\frac{1}{a^{3}}\left[(1-\sigma)\left(\frac{1-\mu^{2}}{\rho} \frac{\partial}{\partial \mu}-\mu \frac{\partial}{\partial \rho}\right) \nabla^{2} \chi+\frac{\partial^{2}}{\partial \rho^{2}}\left(\mu \frac{\partial}{\partial \rho}+\frac{1-\mu^{2}}{\rho} \frac{\partial}{\partial \mu}\right) \chi\right. \\
\left.\quad+\left(\mu \frac{\partial}{\partial \rho}+\frac{1-\mu^{2}}{\rho} \frac{\partial}{\partial \mu}\right)\left(\frac{1}{\rho} \frac{\partial}{\partial \rho}-\frac{\mu}{\rho^{2}} \frac{\partial}{\partial \mu}\right) \chi\right]_{\lambda, 0} \\
=T-T \sum_{n=0}^{\infty}\left\{\frac{(2 n+1) A_{2 n}+(2 n-1+2 \sigma) B_{2 n}}{\lambda^{2 n+3}}\right. \\
\left.\quad-4 n^{2} \lambda^{2 n-2} C_{2 n}-2\left(2 n^{2}+8 n-4 n \sigma+7-5 \sigma\right) \lambda^{2 n} D_{2 n}\right\} P_{2 n+1}^{\prime}(0),
\end{array}
$$

where

$$
P_{2 n+1}^{\prime}(0)=(-1)^{n}\left(\begin{array}{c}
2 n \\
n
\end{array}\right) \frac{2 n+1}{2^{2 n}} .
$$

The following values are obtained.

\begin{tabular}{c|ccc}
$\lambda$ & 0, & $\frac{1}{4}$, & $\frac{1}{2}$ \\
\hline$T^{-1}\left(\max \sigma_{\phi}\right)$ & 2.045, & 2.081, & 2.359
\end{tabular}

The value in the limiting case $\lambda=0$ is the known result of a large tension member having a spherical cavity, that is $(27-15 \sigma) /(14-10 \sigma)$.

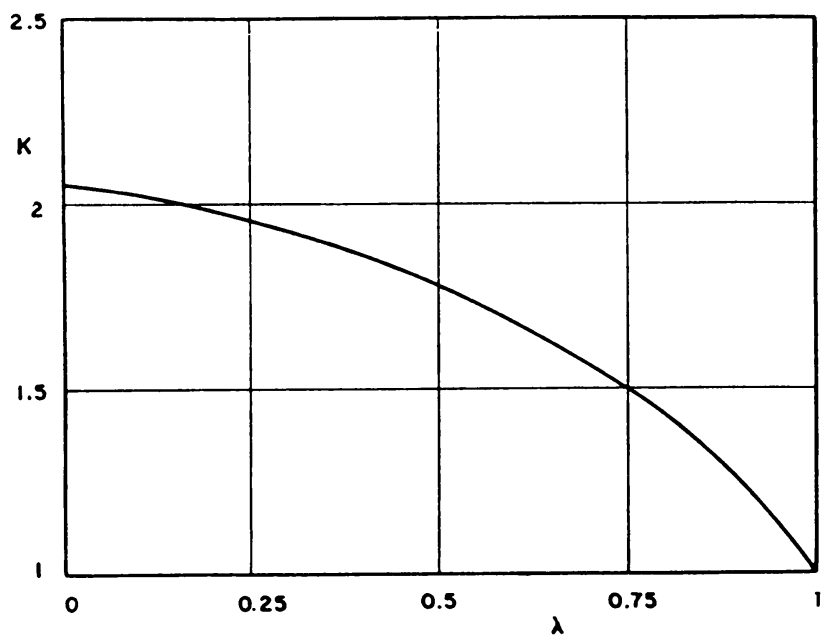

FIG. 2. Stress concentration factor $K$ versus $\lambda$. 
If we define a stress concentration factor $K$ as the ratio of the maximum stress across the minimum section of the cylinder to the mean stress across the same section, then

$$
K=\left(1-\lambda^{2}\right) T^{-1}\left(\max \sigma_{\phi}\right) .
$$

Consequently, we have

\begin{tabular}{c|cccc}
$\lambda$ & 0, & $\frac{1}{4}$, & $\frac{1}{2}$, & 1 \\
\hline$K$ & 2.045, & 1.951, & 1.769, & 1
\end{tabular}

Here the value in the limiting case $\lambda=1$ can be visualized readily from physical consideration of the cylinder. Figure 2 shows the results graphically.

The writer wishes to thank Mr. T. C. Lee for his invaluable assistance in preparing the manuscript. 